\title{
Delirium e demência no idoso: existem fatores de risco comuns?
}

Delirium and dementia in the elderly: are there common risk factors?

\author{
PRISCILla WaCKeR ${ }^{1}$ \\ Paula V. Nunes ${ }^{1}$ \\ ORESTES V. FORLENZA ${ }^{2}$
}

\begin{abstract}
Resumo
Delirium é uma síndrome neurocomportamental decorrente da quebra transitória da homeostase cerebral. Deve-se, invariavelmente, a perturbações sistêmicas ou do sistema nervoso central. No contexto médico geral, a ocorrência de delirium está associada à maior incidência de complicações clínicocirúrgicas, maior tempo de permanência hospitalar e pior recuperação funcional. $\mathrm{O}$ envelhecimento e o comprometimento cognitivo são fatores de risco conhecidos para o delirium. Em pacientes idosos, a ocorrência aumenta o reconhecimento de quadros demenciais preexistentes. Em contrapartida, indivíduos idosos não-demenciados que desenvolvem um episódio de delirium durante uma dada internação hospitalar têm incidência aumentada de demência na evolução em longo prazo. Estudos prospectivos e controlados demonstraram uma associação significativa entre o diagnóstico prévio de delirium e o ulterior desenvolvimento de síndromes demenciais. Essa associação parece ser ainda maior em indivíduos muito idosos. O presente estudo de revisão aborda criticamente a natureza da associação entre delirium e demência. Hipotetiza-se que a sua ocorrência em indivíduos idosos cognitivamente preservados é fator preditivo de deterioração cognitiva, e de risco aumentado para o desenvolvimento de demência, no seguimento de longo termo. As bases neurobiológicas da associação entre delirium e demência são discutidas à luz da hipótese colinérgica da doença de Alzheimer, uma vez que o comprometimento da neurotransmissão colinérgica é denominador comum a essas duas perturbações neuropsíquicas.
\end{abstract}

Palavras-chave: Delirium, demência, doença de Alzheimer, transtorno cognitivo, fatores de risco.

1 Médica psiquiatra. Pós-graduanda pelo Departamento de Psiquiatria do Hospital das Clínicas da Faculdade de Medicina da Universidade de São Paulo (IPq-HC-FMUSP). Pesquisadora do Laboratório de Neurociências (LIM-27) do IPq-HC-FMUSP.

2 Médico psiquiatra. Doutor em Medicina pelo Departamento de Psiquiatria do Hospital das Clínicas da Faculdade de Medicina da Universidade de São Paulo (IPq-HC-FMUSP). Médico Pesquisador e Coordenador do Ambulatório de Transtornos da Memória do LIM-27, IPq-HCFMUSP.

Endereço para correspondência: Priscilla Wacker. Laboratório de Neurociências - LIM 27, Instituto de Psiquiatria do Hospital das Clínicas da Faculdade de Medicina da Universidade de São Paulo, Av. Dr. Ovídio Pires de Campos, 785 - 05403-010 - São Paulo - SP; e-mail: p_wacker@ig.com.br 


\begin{abstract}
Delirium is a neurobehavioral syndrome caused by the transient disruption of cerebral homeostasis, which is invariably subsequent to systemic or central nervous system insults. In general medical settings, the occurrence of delirium is also associated with a higher incidence of post-operative complications, longer hospital stays, and poor functional recovery. Ageing and cognitive decline are known risk factors for the occurrence of delirium. In elderly patients, delirium has been shown to be associated with pre-existing dementia. Conversely, non-demented elderly subjects who develop a new delirium episode display an increased incidence of dementia in the long-term outcome. Prospective case-control studies have demonstrated a significant association between the previous diagnosis of delirium and the development of dementia. The association between delirium and dementia may be even higher among the eldest elderly. After reviewing the relevant literature on the association between delirium and dementia, we hypothesize that the occurrence of delirium in non-demented elderly individuals is predictive of a worse cognitive outcome, and increased risk of dementia, in the long-term follow-up. We further discuss the biological nature of this association in the light of the cholinergic deficit observed in the pathophysiology of both delirium and Alzheimer's disease.
\end{abstract}

Key`words: Delirium, dementia, Alzheimer's disease, cognitive decline, risk factors.

\section{Delirium no contexto clínico e cirúrgico}

O delirium é uma das complicações mais comuns entre pacientes idosos hospitalizados. Estima-se que $20 \%$ a $60 \%$ dos idosos admitidos a serviços de emergência clínica ou cirúrgica apresentem delirium (Erkinjuntti et al., 1986; Gustafson et al., 1988; Francis et al., 1990; Rockwood, 1993; Clayer e Bruckner, 2000; Magaziner et al., 2000; Duppils e Wikblad, 2000; Galanakis et al., 2001; Marcantonio et al., 2001; Gruber-Baldini et al., 2003; Lundström et al., 2003).

Diferentes estudos abordaram a morbimortalidade de pacientes que apresentaram delirium. Levkoff et al. (1992) observaram taxas de mortalidade duas vezes maiores $(26,4 \%)$ entre os indivíduos que tiveram delirium durante suas respectivas internações hospitalares, em seis meses de acompanhamento. Também em seguimento de seis meses, Murray et al. (1993) encontraram sinais de deterioração física em mais da metade dos pacientes que tiveram delirium, ou seja, o dobro do prejuízo evidenciado em pacientes sem tal antecedente. O'Keeffe e Lavan (1997) identificaram uma associação direta entre a ocorrência de delirium e o tempo de permanência hospitalar, além de maiores taxas de admissão em unidades de terapia intensiva (36\% contra 13\%). Mais recentemente, Cole et al. (2003) verificaram que $44 \%$ dos pacientes que apresentaram delirium durante uma dada internação hospitalar evoluíram, ao longo de um seguimento de
12 meses após a alta, com deterioração física ou mental, necessitando de institucionalização.

No contexto das cirurgias ortopédicas, observouse que a ocorrência de delirium aumenta o risco de complicações pós-operatórias e prolonga o período de recuperação, aumentando o tempo de hospitalização (Rogers et al., 1989). Delirium ocorre em até 65\% dos pacientes idosos submetidos à cirurgia do quadril, além de associar-se como fator independente à pior recuperação funcional (Marcantonio, et al., 2001; Gustafon et al., 1988; Gruber-Baldini et al., 2003; Lundström et al., 2003). Estados confusionais foram observados em $40,5 \%$ dos indivíduos operados por fratura de colo de fêmur e em 14,7\% daqueles submetidos à artroplastia (Galanakis et al., 2001).

\section{Fatores de risco}

Delirium é uma síndrome multifatorial e vários fatores de risco exercem efeito no desencadeamento desta desordem neuropsíquica (Francis e Kapoor, 1990). Em contextos clínicos, os fatores mais comumente associados são: (1) idade avançada; (2) presença de doença clínica com comprometimento do estado geral de saúde (Millar, 1981; Williams et al., 1985a; Rockwood, 1989; Francis et al., 1990; Levkoff et al., 1992); (3) presença de demência ou outros acometimentos do sistema nervoso central (SNC) (Erkinjuntti et al., 1986; Gustafson et al., 1988; Schor et al., 1992); (4) polifar- 
macoterapia, particularmente se, entre as múltiplas medicações em uso, incluem-se drogas com ação anticolinérgica, anti-histamínica, sedativo-hipnóticos ou narcóticos, que conferem risco duas a três vezes maior para o desenvolvimento do delirium (Seymour e Pringle, 1983; Leipzig et al., 1987; Cantu e Korek, 1991).

Estudos realizados em contextos cirúrgicos sugeriram que não é o procedimento cirúrgico em si que leva ao aumento no risco de delirium (Schor et al., 1992), e sim os fatores clínicos, hemodinâmicos e farmacológicos associados. Entre eles, desidratação (Levkoff et al., 1986), hipotensão peri-operatória, baixo débito cardíaco, hipóxia no pós-operatório e o uso de medicações com propriedades anticolinérgicas (Millar, 1981; Seymour et al., 1983; Williams et al., 1985b; Gustafson et al., 1988; Schor et al., 1992; Han et al., 2001). O tipo de anestesia (geral ou bloqueio raquimedular) parece não influenciar na incidência do delirium peri-operatório (Riis et al., 1983; Ghoneim, et al., 1988; Warren et al., 1990). A hipoalbuminemia é um achado freqüente em pacientes com delirium (Levkoff et al., 1988; Dickson, 1991). Sua relação com o risco de delirium pode ser indireta, uma vez que sinaliza a ocorrência de desnutrição ou doenças mais graves, que responderiam pelo risco mais expressivo. Alternativamente, a hipoalbuminemia pode afetar a farmacocinética das medicações utilizadas, por permitir menor disponibilidade de ligação protéica, aumentando assim o risco de toxicidade em SNC (Trzepacz et al., 1990). Litaker et al. (2000) observaram a relação entre determinadas variáveis clínicas e o risco de desenvolvimento de delirium em pacientes submetidos a cirurgias eletivas. Destacaram: a história prévia de delirium, a idade avançada (maior ou igual a 70 anos), a preexistência de comprometimento cognitivo, a ocorrência de limitações funcionais no pré-operatório. Consumo excessivo de álcool e o uso de analgésicos opiáceos antes da admissão hospitalar também aumentaram o seu risco.

Doenças psiquiátricas conferem maior risco para o desenvolvimento do delirium, possivelmente em parte pela utilização de medicamentos com ação central (Morse e Lirin, 1969; Gustafson et al., 1988; Folks et al., 1988). Outras condições que parecem estar associadas são: privação de sono, prejuízo sensorial, incontinência urinária e fecal e mudanças ambientais (Levkoff et al., 1986).

\section{Fisiopatologia do delirium}

O delirium é, portanto, visto como uma perturbação das funções neurais decorrente de distúrbios sistêmicos (Lipowski, 1989). Trata-se, por conseguinte, de distúrbio reversível de base metabólica. A sua fisiopatologia ainda é pouco entendida, mas sabe-se que existe envolvimento das vias neurais dependentes da transmissão dopaminérgica, serotoninérgica, colinérgica e histaminérgica (Brown, 2000).
Adicionalmente, a atividade neural depende da reserva de substratos para o metabolismo aeróbio, tais como oxigênio, glicose e fosfatos. Anormalidades do metabolismo oxidativo comprometem a manutenção dos gradientes iônicos, alterando os potenciais elétricos e a repolarização da membrana neuronal, levando à depressão da atividade cerebral. A síntese de neurotransmissores e a depuração de neurotoxinas são também prejudicadas (Francis e Kapoor, 1990; Baethmann e Kempski, 1992).

Os neurônios mais sensíveis a tais insultos são predominantemente os colinérgicos, histaminérgicos e monoaminérgicos (Nicoll et al., 1990). Desse modo, postula-se que condições que levem ao comprometimento das vias colinérgicas e histaminérgicas exerçam papel preponderante no desencadeamento do delirium (Golinger et al., 1987; Trzepacz et al. 1995; Winawer, 2001).

Os neurônios colinérgicos perfazem $25 \%$ das células cerebrais e estão particularmente envolvidos nos processos cognitivos e atencionais. A acetilcolina é o principal modulador das funções dos neurônios corticais e hipocampais. Além dos efeitos decorrentes da baixa disponibilidade sináptica de acetilcolina, no nível do primeiro mensageiro, a disfunção colinérgica está associada ao comprometimento da transdução de sinais no nível pós-sináptico (segundos e terceiros mensageiros), resultando em prejuízos ao metabolismo neuronal e na regulação gênica. Na hipóxia, tanto a síntese quanto a liberação de acetilcolina estão prejudicadas (Gibson e Blass, 1976).

Hall e Popkin (1977) e Gollinger et al. (1987) observaram associação entre a ação de substâncias anticolinérgicas e o delirium. Mais recentemente, em um estudo transversal com 67 idosos portadores de doenças sistêmicas agudas, Flacker et al. (1998) relataram uma associação positiva entre as concentrações séricas de substâncias anticolinérgicas e a ocorrência do delirium.

Outros estudos relatam que episódios de delirium associados a diferentes etiologias têm em comum a atividade colinérgica diminuída na formação reticular (Lipowski, 1990; Flacker et al., 1998; Tune e Egeli, 1999). Durante o estresse oxidativo, a diminuição de síntese e liberação da acetilcolina são anteriores às demais alterações no metabolismo neuronal (Itil e Fink, 1966; Park et al., 1987). Em última análise, tais disfunções, envolvendo neurônios colinérgicos, resultam em alterações cognitivas e atencionais (Ashe e Weinberger, 1991).

A histamina é outro importante modulador das funções neurais. Tanto receptores histamínicos do tipo $\mathrm{H} 1$ quanto $\mathrm{H} 2$ alteram a polarização e a homeostase elétrica dos neurônios hipocampais e corticais (Schwartz et al., 1991). Portanto, a ação de drogas antihistamínicas pode levar ao delirium (White e Rumbold, 1988). Por outro lado, em condições associadas ao excesso de liberação de histamina, tais como no estresse 
cirúrgico e na hipóxia, ocorre indução de mecanismos de apoptose neuronal (Adachi et al., 1991, Fujitani et al., 1996). Assim, acredita-se que tanto o excesso quanto a deficiência de histamina podem estar envolvidos na fisiopatologia do delirium (Brown e Stoudemire, 1989).

A hipóxia pode também levar ao aumento da liberação e à diminuição da recaptura da dopamina (Flacker e Lipsitz, 1999). Sabe-se que a hiperestimulação dopaminérgica pode exercer efeitos tóxicos ao metabolismo neuronal, pela formação de radicais livres, bem como alterar sua função, através da potencialização da atividade glutamatérgica (Pellegrini-Giampietro et al., 1990). O glutamato exerce ação central na lesão neuronal mediada por cálcio (excitotoxicidade), mas não é suficiente para precipitar a lesão sem a ação concomitante da dopamina (Globus et al., 1990).

Outro mecanismo proposto para a fisiopatologia do delirium envolve os níveis de cortisol. Aumentos agudos (muitas vezes iatrogênicos) do cortisol levam a disfunções hipocampais transitórias. Isso é compatível com o fato de que doenças crônicas que cursam com hipercortisolemia, como, por exemplo, a doença de Cushing, levam à redução do volume hipocampal (Starkman et al., 1992; Kiraly et al., 1997). Esse processo está associado a disfunções mitocondriais e a mecanismos de morte neuronal por apoptose (Zamzami et al., 1995; Demonacos et al., 1996). O aumento de cortisol basal é comum no período pós-operatório (Dahanukar et al., 1996). Além disso, sugere-se que pacientes com alterações mitocondriais prévias teriam maior predisposição a desenvolver delirium (Hashimoto et al., 1997).

\section{Relação entre delirium e demência}

Evidências de disfunção colinérgica na doença de Alzheimer foram demonstradas há mais de 20 anos, através de estudos necroscópicos, observando-se redução da atividade das enzimas colina-acetiltransferase (ChAT) e acetilcolinesterase (AChE), respectivamente responsáveis pela síntese e degradação da acetilcolina, em núcleos colinégicos do prosencéfalo basal (Davies e Maloney, 1976). Em seguida, tais achados foram associados ao grau de acometimento cognitivo e à presença de placas senis e emaranhados neurofibrilares em tecidos cerebrais (Wilcock et al., 1982; Mountjoy et al., 1983). Associações altamente significativas foram estabelecidas entre a redução da atividade da ChAT cortical, a contagem de placas senis e os escores dos testes psicométricos
(Perry et al., 1977). Quanto maior o comprometimento cognitivo no ano do óbito, maior a densidade de placas senis e menor a atividade colinérgica global.

Formou-se, assim, a base de sustentação para a hipótese colinérgica da doença de Alzheimer. Desta hipótese decorre a valorização do papel da acetilcolina cortical no comportamento e, sobretudo, na memória, e nela se apóiam os fundamentos da terapia de reposição colinérgica na doença de Alzheimer (Bartus et al., 1982).

A influência dos mecanismos colinérgicos na fisiopatologia do delirium já foi caracterizada (acima). $\mathrm{Na}$ literatura recente, drogas de ação colinérgica foram utilizadas com êxito no manejo clínico do delirium em pacientes idosos. Por exemplo, Wengel et al. (1999) descreveram caso de paciente de 76 anos portador de doença de Alzheimer e submetido à artroplastia de joelho, em que o delirium pós-operatório foi revertido mediante o tratamento com o inibidor da acetilcolinesterase cloridrato de donepezil. Fisher (2001) relatou o caso de uma paciente de 70 anos que apresentou delirium pela intoxicação pelo lítio, e que obteve recuperação completa do quadro confusional mediante tratamento com rivastigmina.

Uma vez que a doença de Alzheimer e o delirium têm na disfunção colinérgica mecanismo etiopatogênico comum, hipotetiza-se que os indivíduos com demência são particularmente suscetíveis ao delirium. De fato, estudos disponíveis na literatura mostram que a constatação de episódio de delirium aumenta a sensibilidade para o diagnóstico de demência já existente (Rockwood et al., 1999; Rahkonen et al., 2001). Esses autores realizaram estudos prospectivos com indivíduos idosos portadores de doenças sistêmicas, agrupados segundo a ocorrência ou não de delirium, e observaram maior risco de evolução para demência entre aqueles que apresentaram um ou mais episódios de delirium.

Recentemente, Lundström et al. (2003) avaliaram as funções cognitivas de pacientes submetidos a artroplastias de quadril, devido a fraturas de colo de fêmur, também classificados segundo a ocorrência ou não de delirium pós-operatório. Os grupos foram seguidos prospectivamente por período de quatro anos, constatando-se maior incidência de síndromes demenciais (69\%) entre os indivíduos que apresentaram delirium, contra a incidência de $20 \%$ entre aqueles que não o apresentaram. Isso mostra que a sua ocorrência em indivíduos cognitivamente intactos sinaliza maior vulnerabilidade para a demência. 
Referências bibliográficas

AdachI, N.; OISHI, R.; SAekI, K. - Changes in the metabolism of histamine and monoamines after occlusion of the middle cerebral artery in rats. $J$ Neurochem 57:61-6, 1991.

AsHE, J.H.;WEINBERGER, N.M. - Acetylcoline modulation of cellular excitability via muscarinic receptors: functional plasticity in auditory cortex. In: Richardson, R.T. (ed.) Activation to Acquisition: Functional Aspects of the Basal Forebrain Cholinergic System, Birkhauser, Boston, pp.189-246, 1991.

Baethmann, A.; KempSKI, 0. - Biochemical factors and mechanisms of secondary brain damage in cerebral ischemia and trauma. In: Bazan, N.; Braquet, P.; Ginsberg, M. (eds.) Neurochemical Correlates of Cerebral Ischemia, Plenum Press, New York, pp.71-98, 1992.

Bartus, R.T.; Dean, R.L.; Beer, B.; LIPPA, A.S. - Cholinergic hypothesis of geriatric memory dysfunction: a critical review. Science 217:408-17, 1982.

Brown, T.M.; Stoudemire, A. - Antipsychotics in Psychiatric Side Effects of Prescription and Over-the-Counter Medications, American Psychiatric Press, Washington, D.C., pp.3-33, 1989.

Brown, T.M. - Basic Mechanisms in the pathogenesis of Delirium. In: Stoudemire, A.; Fogel, B.; Greenberg, D. (eds). Psychiatric care of the medical patients, Oxford University Press, New York, pp.571-80, 2000.

Cantu, T.G.; KoreK, J.S. - Central nervous system reactions to histamine-2 receptor blockers. Ann Intern Med 114:102734, 1991.

Clayer, M.; Bruckner, J. - Occult Hypoxia After Femoral Neck Fracture and Elective Hip Surgery. Clin Orthop 370:26571, 2000.

Cole, M.; McCusker, J.; Dendukurl, N.; Han, L. - The Prognostic Significance of Subsyndromal Delirium in Elderly Medical Inpatients. J Am Geriatr Soc 51:754-60, 2003.

Dahanukar, S.A.; Thatte, U.M.; Desmukh, U.D.; KulKarni, M.K.; Bapat, R.D. - The influence of surgical stress on the psychoneuroendocrine-immune axis. J Postgrad Med 42:12-4, 1996.

Davies, P.; Maloney, A.J.F. - Selective loss of cholinergic neurons in Alzheimer's disease. Lancet 2:1403, 1976.

Demonacos, C.V.; Karayanni, N.; Hatzoglou, E. - Mitochondrial genes as sites of primary action of steroid hormones. Steroids 61:226-32, 1996.

Dickson, L.R. - Hypoalbuminemia in delirium. Psycosomatics 32:317-23, 1991.

DuppILS, G.S.; WIKBLAD, K. - Acute Confusional states in patients undergoing hip surgery. A prospective observation study. Gerontology 46(1):28-35, 2000.

ErkinjunttI, T.; WiKstrom, J.; Palo, J. et al. - Dementia among medical inpatients: Evaluation of 2000 consecutive admissions. Arch Intern Med 146: 1923-6, 1986.

FISCHER, M.D. - Successful treatment of nonanticholinergic delirium with a cholinesterase inhibitor. J Clin Psychopharmacol 21(1):118, 2001.
Flacker, J.M.; Cummings, V.; Mach, J.R.; Bettin, K.; Kiely, D.K.; WEI, J. - The Associations of serum anticholinergic activity with delirium in elderly medical patients. $A m \mathrm{~J}$ Geriatr Psychiatry 134:31-41, 1998.

FLACKER, J.M.; LIPSITZ, L.A. - Neural mechanisms of delirium: current hypotheses and evolving concepts. J Gerontol A Biol Sci Med Sci 54(7):B275, 1999.

Folks, D.G.; Freeman, A.M.; SokoL, R.S. et al. - Cognitive dysfunction after coronary artery bypass surgery: $A$ case-control study. South Med J 81:202-6, 1988.

Francis, J.; KAPOOR, W.N. - Delirium in hospitalized elderly. J Gen Intern Med 5:65-79, 1990.

Francis, J.; Martin, D.; Kapoor, W.N. - A prospective study of delirium in hospitalized elderly. JAMA 263:1097-110, 1990.

FuJitani, T.; Adachi, N.; Nagaro, T. et al. - Histaminergic H2 action protects hippocampal CA1 neurons by prolonging the onset of the anoxic depolarization in gerbils. J Neurochem 67:2613-5, 1996.

GalanAkis, P.; FörstL, H. et al. - Acute confusional state in the elderly following hip surgery: incidence, risk factors and complications. Int Geriatr Psychiatry 16:349-55, 2001.

GHoneIm, M.M.; JAMES, V.; HinRICHS,D. et al. - Comparison of Psycologic and Cognitive Functions after General or Regional Anesthesia. Anestesiology 69:507-15, 1988.

GIBSON, G.; BLASS, J. - Impaired synthesis of acetylcoline in brain acompanying mild hypoxia and hypoglycemia. J Neurochem 27:37-42, 1976.

Globus, M.; B usto, R.; DietRich, W. et al. - Excessive release of glutamate is not sufficient for the development of ischemic damage in vivo. Stroke 21:176, 1990.

Golinger, R.; PeEt, T.; TUNE, L. - Association of elevated plasma anticholinergic activity with delirium in surgical patientes. Am J Psychiatry 144:1218-20, 1987.

Gruber-Baldin, A.L.; Zimmerman, S.; Morrison, S. et al. - Cognitive Impairment in Hip Fracture Patients: Timing of Detection and Longitudinal Follow-Up. J Am Geriatr Soc 51:1227-36, 2003.

Gustafson, Y.; Berggren, D.; Brannstrom, B. et al. - Acute confusional states in elderly patients treated for femoral neck fracture. J Am Geriat Soc 36:525-30, 1988.

HaLL, R.; PoPKIN, H.L. - Angel's trumpet psychosis: a central nervous system anticholinergic syndrome. Am $J$ Psychiatry 134: 312-4, 1977.

Han, L.; McCusker, J.; Cole, M.; Abrahamowick, M.; Primeau, F.; Élie, M. - Use of Medications with Anticholinergic Effect Predicts Clinical severity of delirium Symptons in older Medical Inpatients. Arch intern Med 161:1099-105, 2001.

Hashimoto, H.; NaKamura, K.; IzUmirama, N. - Postoperative delirium easily develops in patients with intramithocondrial inclusion bodies in colonic neurons. Aging (Milano) 9:180-4, 1997.

ITIL, T.; FINK, M. - Anticholinergic drug-induced delirium: Experimental modification, quantitative EEG and behavioral correlations. J New Ment Dis 6:492-507, 1966. 
Kiraly, S.J.; AncilL, R.J.; Dimitrova, G. - The relationship of endogenous cortisol to psychiatric disorder: a rewiew. Can J Psychistry 42:415-20, 1997.

LEIPZIG, R.M.; Goodman, H.; G ray, G. et al. - Reversible, narcoticassociated mental status impairment in patients with metastatic cancer. Pharmacology 35:47-54, 1987.

LevkofF, S.E.; Besdine, R.W.; WetLe, T. - Acute confusional state (delirium) in the hospitalized elderly. Ann Rev Gerontol Geriatr 6:1-26, 1986.

LevKoff, S.E.; Safran, C.; Clearly, P.D. etal. - Identification of factors associated with the diagnosis of delirium in elderly hospitalized patients. J Am Geriatr Soc 36:1099-104, 1988.

Levkoff, S.E.; Evans, D.A.; Liptzin, B. et al. - Delirium the occurrence and persistence of symptons among elderly hospitalized patientes. Arch Intern Med 152:334-40, 1992.

LIPowskI, Z.J. - Delirium in the elderly patient. N Engl J Méd 320:578-82, 1989.

LIPowskı, Z.J. - Delirium: acute confusional states, Oxford Univ. press, New York, pp. 229-76, 1990.

Litaker, D.; Locala, J.; Franco, K.; Bronsom, D.L.; Tannous, Z. Preoperative risk factors for postoperative delirium. Int Psychogeriatr 12(4):473-81, 2000.

Lundström, M.; Edlund, A.; Bucht, G.; Karlsson, S.; Gustafson, Y. Dementia after Delirium in Patients with Femoral Neck Fractures. J Am Geriatr Soc 51: 1002-6, 2003.

Magaziner, J.; Hawkes, W.; Hebel, J.R. et al. - Recovery from hip fracture in eight areas of function. $J$ Gerontol $A$ Biol Sci Med Sci55A:498-m507, 2000.

Marcantonio, E.R.; Flacker, J.M.; Wright, R.J.; Resnick, N.M. Reducing delirium after hip fracture: a randomized trial. J Am Geriatr Soc 49(5):516-22, 2001.

MILLAR, H.R. - Psychiatric morbidity in elderly surgical patientes. Br L Psychiatry 138:17-20, 1981.

MorSE, R.M.; Litin, E.M. - Postoperative delirium: A study of etiologic factors. Am J Psychiatry 126:136-43, 1969.

Mountuoy, C.Q.; Roth, M.; Evans, N.J.R.; Evans, H.M. - Cortical neuronal counts in normal elderly controls and demented patients. Neurobiol. Aging 4:1-11, 1983.

MurraY, A.M.; Levkoff, S.E.; WetLE, T.T. - Acute delirium and functional decline in the hospitalized in the hospitalized elderly patient. J Gerontol 48:M181-6, 1993.

Nicoll, R.; MalenKa, R.; KaUer, J. - Functional comparison of neurotransmitter receptor subtypes in mammalian central nervous system. Physiol Rev 70:513-65, 1990.

O'KeefFe, S.; Lavan, J. - The prognostic significance of delirium in older hospital patients. JAm Geriatr Soc 45:174-8, 1997.

PaRK, I.;THORN, M.; B ACHELARD, H. - Threshold requirements for oxygen in the release of acetyilcoline from, and in the maintance of the energy state in rat synaptosomes. $J$ Neurochem 49:781-8, 1987.

Pellegrini-Giampietro, D.; Cherici, G.; Alesiani, M. et al. Excitatory amino acid release and free radical formation may cooperate in the genesis of ischemiainduced neuronal damage. J Neurosci 10:1035-41, 1990.

Perry, E.; Perry, R.H.; Blessed, G.; Tomlinson, B.E. - Necropsy evidence of central cholinergic deficits in senile dementia. Lancet 1:189, 1977.
Rahkonen, T.; Eloniemi-Sulkava, U.; Halonen, P.; Verkkoniemi, A.; NıInISTo, L.; NotKoLA, I.L. - Delirium in the non-demented oldest old in the general population: risk factors and prognosis. Int J Geriatr Psychiatry 16(4):415-21, 2001.

RIIS, J.; LOMHOLT, B.; HAXHOLDT, O. et al. - Immediate and long-term mental recovery from general versus epidural anesthesia in elderly patients. Acta Anaesthesiol Scand 27:44-9, 1983.

Rockwood, K. - Acute confusion in elderly medical patients. $J$ Am Geriatr Soc 37:150-4, 1989.

RockW00D, K. - The ocurrence and duration of symptoms in elderly patients in delirium. J Gerontol 48:M162-6, 1993.

Rockwood, K.; Cosway, S.; Carver, D. et al. - The risk of dementia and death after delirium. Age Ageing 28:551-6, 1999.

Rogers, M.P.; LIANG, M.H.; DaLtROY, L.H. et al. - Delirium after elective orthopedic surgery: risk factors and natural history. Int J Psychiatry Med 19:109-21, 1989.

Schwartz, J.C.; Arrang, J.M.; Garbarg, M. et al. - Histaminergic transmission in the mammalian brain. Physiol Rev71:1-51,1991.

SchoR, J.D.; LEVKOFF, S.E.; LIPSITZ, L.A. et al. - Risk factors for delirium in hospitalized elderly. JAMA 267:827-31, 1992.

Seymour, D.G.; Pringle, R. - Post-operative complications in the elderly surgical patients. Gerontology29:262-70, 1983.

StaRKMAN, M.N.;GeBARSK,S.S.;B RENST, S. etal.- Hippocampal formation volume, memory disfunction, and cortisol levels in patients with Cushing's syndrome. Biol Psychiatry32:756-65, 1992.

Trzepacz, P.; Brown, T.M.; Stoudemire, A. - Substance-induced delirium. In: Gabbard, G.0. (ed.) Treatments of Psychiatric Disorder, vol.1. American Psychiatric Press, Washington, D.C., pp.445-78, 1995.

Trzepacz, P.T.; Francis, J. - Low serum albunim and risk of delirium (letter). Am J Psychiatry 147:675, 1990.

Tune, L.E.; EgEL, S. - Acetylcholine and delirium. Dement. Geriatr. Cogn. Disord. 10:342-4, 1999.

Warren, R.; Nielson, D.; Adrian, W. et al. - Long-term Cognitive and Social Sequelae of General versus Regional Anesthesia during Arthroplasty in the elderly. Anestesiology 73:1103-9, 1990.

Wengel, S.P.; Burke, W.J.; Roccaforte, W.H. - Donepezil for postoperative delirium associated with Alzheimer's disease. J Am Geriatr Soc, 47(3):379-380, 1999.

WHITE, J.M.; RUMBOLD, G.R. - Behavioural effects of histamine and its antagonists: a review. Psychopharmacology 95:1-14,1988.

WILCOCK, G.K.; EsIRI, M.M.; BowEN, D.M.; SMITH, C.T.T. - Alzheimer's disease: correlation of cortical choline acetyltransferase with the severity of dementia and histological abnormalities. J. Neurol. Sci. v.57, p.407-17, 1982.

Williams, M.A.; Campbell, E.B.; Raynor, W.J. et al. - Reducing acute confusional states in elderly patients with hip fractures. Res Nurs Health 8:329-37,1985a.

Williams, M.A.; Campbell, E.B.; RaynOR, W.J. et al. - Predictors of acute confusional states in hospitalized elderly patients. Res Nurs Health 8:31-40, 1985b.

Winawer, N. - Postoperative delirium. Med Clin North Am 85(5):1229, 2001.

Zamzami, N.; Marchetti, P.; Castedo, M. et al. - Sequential reduction of mitochondrial transmembrane potential and generation of reactive oxygen species in early programmed cell death. J Exp Med 182:367-77, 1995. 